\title{
Three fundamental masses derived by dimensional analysis
}

\author{
Dimitar Valev \\ Stara Zagora Department, Space and Solar-Terrestrial Research Institute, \\ Bulgarian Academy of Sciences, P.O. Box 73, 6000 Stara Zagora, Bulgaria
}

\begin{abstract}
Three new mass dimension quantities have been derived by dimensional analysis, in addition to the famous Planck mass $m_{P} \approx 2.17 \times 10^{-8} \mathrm{~kg}$. These masses have been derived by means of fundamental constants - the speed of light $(c)$, the gravitational constant $(G)$, the Plank constant $(\hbar)$ and the Hubble constant $(H)$. The enormous mass $m_{1} \sim c^{3} /(G H) \sim 10^{53} \mathrm{~kg}$ practically coincides with the Hoyle-Carvalho formula for the mass of the observable universe. The extremely small mass $\mathrm{m}_{2} \sim(\hbar H) / \mathrm{c}^{2} \sim 10^{-33} \mathrm{eV}$ has been identified with the Hubble mass, which seems close to the graviton mass. It is noteworthy that the Planck mass appears geometric mean of the masses $m_{1}$ and $m_{2}$. The mass $m_{3} \sim\left[\left(H \hbar^{3}\right) / G^{2}\right]^{1 / 5} \sim 10^{7} \mathrm{GeV}$ could not be unambiguously identified at present time. Besides, the order of magnitude of the total density of the universe has been estimated by this approach.
\end{abstract}

Key words: Planck mass, dimensional analysis, mass of the universe, Hubble mass, graviton mass

PACS numbers: 06.20.fa, 06.30.Dr, 14.70.Kv, 98.80.Es

\section{Introduction}

The Planck mass $m_{P} \sim \sqrt{\frac{\hbar c}{G}}$ has been introduced in [1] by means of three fundamental constants - the speed of light in vacuum $(c)$, the gravitational constant $(G)$ and the reduced Plank constant $(\hbar)$. Since the constants $c, G$ and $\hbar$ represent three very basic aspects of the universe (i.e. the relativistic, gravitational and quantum phenomena), the Plank mass appears to a certain degree a unification of these phenomena. The Plank mass have many important aspects in modern physics. One of them is that the energy equivalent of Planck mass $E_{P}=m_{P} c^{2} \sim \sqrt{\frac{\hbar c^{5}}{G}} \sim 10^{19} \mathrm{GeV}$ appears unification energy of the fundamental interactions [2]. Also, the Planck mass can be approximately derived by setting it as a mass, whose Compton wavelength and Schwartzchild radius are equal [3].

The Plank mass formula has been derived by dimensional analysis using fundamental constants $c, G$ and $\hbar$. The dimensional analysis is a conceptual tool often applied in physics to understand physical situations involving certain physical quantities [4-8]. It is routinely used to check the plausibility of the derived equations and computations. When it is known, the certain quantity with which other determinative quantities would be connected, but the form of this connection is unknown, a dimensional equation is composed for its finding. In the left side of the equation, the unit of this quantity $q_{0}$ with its dimensional exponent has been placed. In the right side of the equation, the product of units of the determinative 
quantities $q_{i}$ rise to the unknown exponents $n_{i}$ has been placed $\left[q_{0}\right] \sim \prod_{i=1}^{n}\left[q_{i}\right]^{n_{i}}$, where $n$ is positive integer and the exponents $n_{i}$ are rational numbers. Most often, the dimensional analysis is applied in the mechanics and other fields of the modern physics, where there are many problems with a few determinative quantities. Many interesting and important problems related to the fundamental constants have been considered in [9-13].

The discovery of the linear relationship between recessional velocity of distant galaxies, and distance $v=H r$ [14] introduces new fundamental constant in physics and cosmology the famous Hubble constant $(H)$. Even seven years before, Friedman [15] derived his equations from the Einstein field equations [16], showing that the universe might expand at a rate calculable by the equations. Hubble constant determines the age of the universe $H^{-1}$, the Hubble distance $c H^{-1}$, the critical density of the universe $\rho_{c}=\frac{3 H^{2}}{8 \pi G}$ [17], and other largescale properties of the universe.

Because of the importance of the Hubble constant, in the present paper we include $H$ in the dimensional analysis together with $c, G$ and $\hbar$ aiming to find the new mass dimension quantities $m_{i} \sim \prod_{j=1}^{3} q_{j}^{n_{j}}$, where every triad $q_{1}, q_{2}, q_{3}$ consists of three constants $c, G, \hbar$ and $H$. Thus, the Hubble constant will represent the cosmological phenomena in new derived fundamental masses. According to the recent cosmology, the Hubble 'constant' slowly decreases with the age of the universe, but there are indications that other constants, especially gravitational and fine structure constants also vary with time [18-20]. That is why, the Hubble constant could deserve being treated on an equal level with the other three constants used by Planck.

\section{Three fundamental masses derived by dimensional analysis}

Below, we obtain a mass dimension quantity $m_{1}$ constructed from the fundamental constants - the speed of light $(c)$, the gravitational constant $(G)$ and the Hubble constant $(H)$ using dimensional analysis. A quantity $m_{1}$ having mass dimension could be constructed by means of the fundamental constants $c, G$ and $H$ :

$$
m_{1}=k c^{n_{1}} G^{n_{2}} H^{n_{3}}
$$

where $n_{1}, n_{2}$ and $n_{3}$ are unknown exponents to be determined by matching the dimensions of both sides of the equation, and $k$ is dimensionless parameter of an order of magnitude of a unit.

As a result we find the system of linear equations:

$$
\begin{aligned}
& n_{1}+3 n_{2}=0 \\
& -n_{1}-2 n_{2}-n_{3}=0 \\
& -n_{2}=1
\end{aligned}
$$

The unique solution of the system is $n_{1}=3, n_{2}=-1, n_{3}=-1$. Replacing obtained values of the exponents in equation (1) we find formula (3) for the mass $m_{1}$ : 


$$
m_{1} \sim \frac{c^{3}}{G H}
$$

First of all, the formula (3) has been derived by dimensional analysis in [21]. This formula practically coincides with the Hoyle formula for the mass of the observable universe $M=\frac{c^{3}}{2 G H}$ [22] and perfectly coincides with Carvalho formula [23] for the mass of the observable universe, obtained by totally different approach.

Evidently, the Hoyle formula coincides with the mass of the Hubble sphere $M_{H}$, i.e. mass of the sphere having radius equal to the Hubble distance $\mathrm{cH}^{-1}$ and density equal to the total density of the universe $\bar{\rho} \approx \rho_{c}$ :

$$
M_{H}=\frac{4}{3} \pi \frac{c^{3}}{H^{3}} \frac{3 H^{2}}{8 \pi G}=\frac{c^{3}}{2 G H}
$$

The recent experimental values of $c, G$ and $H$ are used: $c=299792458 \mathrm{~m} \mathrm{~s}^{-1}, G=$ $6.673 \times 10^{-11} \mathrm{~m}^{3} \mathrm{~kg}^{-1} \mathrm{~s}^{-2}$ [24] and $\mathrm{H} \approx 70 \mathrm{~km} \mathrm{~s}^{-1} \mathrm{Mps}^{-1}$ [25]. Replacing this values in (3) we obtain $m_{1} \sim 1.76 \times 10^{53} \mathrm{~kg}$. Therefore, the enormous mass $m_{1}$ would be identified with the mass of the observable universe.

Analogously, by means of the fundamental constants $c, \hbar$ and $H$, a quantity $m_{2}$ having dimension of a mass could be constructed:

$$
m_{2}=k c^{n_{1}} \hbar^{n_{2}} H^{n_{3}}
$$

We determine the exponents $n_{1}=-2, n_{2}=1, n_{3}=1$ by the dimensional analysis again. Replacing the obtained values of the exponents in equation (5) we find formula (6) for the $\operatorname{mass} m_{2}$ :

$$
m_{2} \sim \frac{\hbar H}{c^{2}}
$$

Replacing the recent values of the constants $c$, $\hbar$ and $H$ in (6) we obtain $m_{2} \sim 2.70 \times 10^{-69}$ $k g=1.52 \times 10^{-33} \mathrm{eV}$. This exceptionally small mass coincides with the so called "Hubble mass" $[26,27]$, which seems close to the graviton mass obtained by different methods [28-31]. The mass $m_{2}$ is in several orders of magnitude smaller than the upper limit of the graviton mass, obtained by astrophysical constraints [32]. From equation (6) we find that the reduced Compton wavelength $\lambda_{2}$ of this mass is equal to the Hubble distance $c H^{-1}$ :

$$
\bar{\lambda}_{2}=\frac{\hbar}{m_{2} c}=c H^{-1} \sim 1.3 \times 10^{26} \mathrm{~m}
$$

From formulae (3) and (6) we find an interesting relation (8): 


$$
\sqrt{m_{1} m_{2}}=\sqrt{\frac{c^{3}}{G H} \frac{\hbar H}{c^{2}}}=\sqrt{\frac{\hbar c}{G}} \equiv m_{P}=2.17 \times 10^{-8} \mathrm{~kg}
$$

Therefore, the Planck mass appears geometric mean of the Hubble mass and the mass of the observable universe. As the physical quantity mass is among the most important properties of the matter, the formula (8) hints at a deep relation of the micro particles and the entire universe.

Besides, the ratios (9) take place:

$$
\frac{m_{1}}{m_{P}}=\frac{m_{P}}{m_{2}}=\frac{c H^{-1}}{r_{P}}=\frac{H^{-1}}{t_{P}}=\sqrt{\frac{c^{5}}{G \hbar H^{2}}} \sim 8 \times 10^{60}
$$

where $r_{P}=\sqrt{\frac{G \hbar}{c^{3}}}$ is the Planck length, $t_{P}$ is the Plank time, $c H^{-1}$ is the Hubble distance, and $H^{-1}$ is the Hubble time.

The third quantity $m_{3}$, having mass dimension could be constructed by means of the fundamental constants $G, \hbar$ and $H$ :

$$
m_{3}=k G^{n_{1}} \hbar^{n_{2}} H^{n_{3}}
$$

We determine the exponents $n_{1}=-\frac{2}{5}, n_{2}=\frac{3}{5}, n_{3}=\frac{1}{5}$ by dimensional analysis again. Replacing the obtained values of the exponents in equation (10) we find formula (11) for the mass $m_{3}$ :

$$
m_{3} \sim \sqrt[5]{\frac{H \hbar^{3}}{G^{2}}}
$$

Replacing the recent values of the constants $G, \hbar$ and $H$, the mass $m_{3}$ takes value $m_{3}$ $1.43 \times 10^{-20} \mathrm{~kg} \approx 8.0 \times 10^{6} \mathrm{GeV}$. This mass is a dozen of orders of magnitude lighter than the Planck mass and several orders of magnitude heavier than the heaviest known particles like the top quark $m_{t} \approx 174.3 \mathrm{GeV}$ [33]. On the other hand, the energy $m_{3} c^{2} \sim 8 \times 10^{6} \mathrm{GeV}$ appears medial for the important GUT scale $E_{G U T} \sim 10^{16} \mathrm{GeV}$ and electroweak scale $E_{E W} \sim 10^{2} \mathrm{GeV}$. Therefore, the mass/energy $m_{3}$ could not be unambiguously identified at the present time, and it could be considered as heuristic prediction of the suggested approach.

Below, we demonstrate the heuristic power of the suggested approach approximately estimating the total density of the universe by dimensional analysis. Actually, a quantity $\rho$ having dimension of density could be constructed by means of the fundamental constants $c, G$ and $H$ :

$$
\rho=k c^{n_{1}} G^{n_{2}} H^{n_{3}}
$$

where $k$ is a dimensionless parameter of the order of magnitude of unit. 
By the dimensional analysis, we have found the exponents $n_{1}=0, n_{2}=-1, n_{3}=2$. Therefore:

$$
\rho \sim \frac{H^{2}}{G} \approx 7.93 \times 10^{-26} \mathrm{~kg} \mathrm{~m}^{-3}
$$

The recent Cosmic Microwave Background $(C M B)$ observations show that the total density of the universe $\bar{\rho}$ is [34-36]:

$$
\bar{\rho}=\Omega \rho_{c} \approx \rho_{c}=\frac{3 H^{2}}{8 \pi G} \sim 10^{-26} \mathrm{~kg} \mathrm{~m}^{-3}
$$

Evidently, the density $\rho$ derived by means of the fundamental constants $c, G$ and $H$ coincides with formula (14) for the total density of the universe with an accuracy of a dimensionless parameter of an order of magnitude of a unit. Besides, the formula (13) could be derived by means of other triad of fundamental constants, namely $G, \hbar$ and $H$.

\section{Conclusions}

Three new mass dimension quantities $m_{i}$ have been derived by dimensional analysis, in addition to the Planck mass $m_{P} \sim \sqrt{\frac{\hbar c}{G}} \sim 2.17 \times 10^{-8} \mathrm{~kg}$. Four fundamental constants - the speed of light in vacuum $(c)$, the gravitational constant $(G)$, the reduced Plank constant $(\hbar)$ and the Hubble constant $(H)$ have been involved in the dimensional analysis. The first derived mass dimension quantity $m_{1} \sim \frac{c^{3}}{G H} \sim 10^{53} \mathrm{~kg}$ practically coincides with the Hoyle-Carvalho formula for the mass of the universe obtained by totally different approach. The exceptionally small mass dimension quantity $m_{2} \sim \frac{\hbar H}{c^{2}} \sim 10^{-33} \mathrm{eV}$ has been identified with the Hubble mass, which seems close to the graviton mass. It is amazing that the Planck mass appears geometric mean of the masses $m_{1}$ and $m_{2}$, i.e. $m_{P}=\sqrt{m_{1} m_{2}}$. The third derived mass $m_{3} \sim \sqrt[5]{\frac{H \hbar^{3}}{G^{2}}} \sim 10^{7}$ $\mathrm{GeV}$ could not be identified unambiguously at present time. The identification of the two derived masses reinforces the trust in the suggested approach.

According to the Big Bang cosmology, the Hubble constant decreases with the age of the universe. Therefore, the mass of the universe $m_{1} \sim \frac{c^{3}}{G H}$ increases, whereas the Hubble mass $m_{2} \sim \frac{\hbar H}{c^{2}}$ and mass $m_{3} \sim \sqrt[5]{\frac{H \hbar^{3}}{G^{2}}}$ decrease with time. Nevertheless, the Planck mass remains a geometric mean of the Hubble mass and mass of the observable universe. 


\section{References}

[1] M. Planck, The Theory of Radiation, Dover Publications, NY (1959) (translated from 1906).

[2] H. Georgi, H. R. Quinn and S. Weinberg, Phys. Rev. Lett. 33, 451 (1974).

[3] P. G. Bergmann, The Riddle of Gravitation, Dover Publications, NY (1992).

[4] P. W. Bridgman, Dimensional Analysis, Yale Univ. Press, Yale (1922).

[5] H. E. Huntley, Dimensional Analysis, Dover Publications, NY (1967).

[6] R. Kurth, Dimensional Analysis and Group Theory in Astrophysics, Pergamon Press, Oxford (1972).

[7 R. Bhaskar and A. Nigam, Artificial Intelligence 45, 73 (1990).

[8] G. W. Petty, Software - Practice and Experience 31, 1067 (2001).

[9] J. M. Levy-Leblond, Riv. Nuovo Cim. 7, 187 (1977).

[10] M. J. Duff, L. B. Okun and G. Veneziano, J. High En. Phys., Issue 03, 023 (2002).

[11] M. J. Duff, arxiv:hep-th/0208093.

[12] J. D. Barrow, The Constants of Nature: From Alpha to Omega, Jonathan Cape, London (2002).

[13] H. Fritzsch, The Fundamental Constants, a Mystery of Physics, World Scientific, Singapore (2009).

[14] E. Hubble, Proc. Nat. Acad. Sci. 15, 168 (1929).

[15] A. Friedman, Z. Physik 10, 377 (1922).

[16] A. Einstein, Annalen der Physik 354, 769 (1916).

[17] P. J. Peebles, Physical Cosmology, Princeton Univ. Press, Princeton (1971).

[18] P. A. M. Dirac, Nature 139, 323 (1937).

[19] Y. Wu and Z. Wang, Phys. Rev. Lett. 57, 1978 (1986).

[20] J. K. Webb et al., Phys. Rev. Lett. 87, 091301 (2001).

[21] D. Valev, Compt. Rend. Acad. Bulg. Sci., Spec. Issue, 233 (2009), arxiv:0909.2726.

[22] F. Hoyle, in Proc. $11^{\text {th }}$ Solvay Conference in Physics, Brussels, 1958, edited by R. Stoops.

[23] J. C. Carvalho, Int. J. Theor. Phys. 34, 2507 (1995).

[24] P. Mohr and B. Taylor, J. Phys. Chem. Ref. Data 28, 1713 (1999).

[25] J. R. Mould et al., Astrophys. J. 529, 786 (2000).

[26] I. Maor and R. Brustein, Phys. Rev. D 67, 103508 (2003).

[27] J. P. Gazeau and F. Toppan, Class. Quantum Grav. 27, 025004 (2010).

[28] J. F. Woodward, R. J. Crowley and W. Yourgrau, Phys. Rev. D 11, 1371 (1975).

[29] S. S. Gershtein, A. A. Logunov and M. A. Mestvirishvili, Dokl. Phys. 43, 293 (1998).

[30] D. Valev, Aerospace Res. Bulg. 22, 68 (2008), arxiv:hep-ph/0507255.

[31] M. E. Alves, O. D. Miranda and de J. C. Araujo, arxiv:0907.5190.

[32] A. S. Goldhaber and M. M. Nietto, Phys. Rev. D 9, 1119 (1974).

[33] M. Mangano and T. Trippe, Europ. Phys. J. C 15, 385 (2000).

[34] A. Balbi et al., Astrophys. J. 545, L1 (2000).

[35] P. de Bernardis et al., Nature 404, 955 (2000).

[36] D. N. Spergel et al., Astrophys. J. Suppl. Series 148, 175 (2003). 